\title{
Microwave Spectrum and Quadrupole Coupling Constants of 2-Bromothiophene
}

\author{
P. J. Mjöberg, W. M. Ralowski, and S. O. Ljunggren \\ Department of Physical Chemistry, The Royal Institute of Technology, S-100 44 Stockholm 70, \\ Sweden
}

(Z. Naturforsch. 30 a, 541-548 [1975] ; received February 8, 1975) The microwave spectra of the two ${ }^{79} \mathrm{Br}$ and ${ }^{81} \mathrm{Br}$ isotopic species of 2 -bromothiophene have been
measured in the region $18000-40000 \mathrm{MHz}$.
For both isotopic species, the rotational constants of the ground state and one vibrationally ex-
cited state were determined, as well as the centrifugal distortion coefficients of the ground state.
The ground state rotational constants in $\mathrm{MHz}$ are as follows:

$$
\begin{array}{lc}
\mathrm{C}_{4} \mathrm{H}_{3}{ }^{32} \mathrm{~S}^{79} \mathrm{Br} & \mathrm{C}_{4} \mathrm{H}_{3}{ }^{32} \mathrm{~S}^{81} \mathrm{Br} \\
A=5403.432 \pm 0.111 & 5403.563 \pm 0.095 \\
B=1139.0689 \pm 0.0010 & 1126.5173 \pm 0.0011 \\
C=940.5142 \pm 0.0018 & 931.9315 \pm 0.0009
\end{array}
$$

In order to perform a second-order perturbation treatment of the quadrupole interaction, the matrix elements of products of direction cosines in terms of the symmetric top wave functions have been derived. By the first- and second-order perturbation analysis of the hyperfine splittings of the rotational lines, the nuclear quadrupole coupling constants have been determined. The values in $\mathrm{MHz}$ are

$$
\begin{aligned}
& \chi_{a a}=592.7 \pm 1.5 \\
& \chi_{b b}=-295.3 \pm 0.6 \\
& \chi_{c c}=-297.4 \pm 1.6 \\
& \chi_{a b}=80 \pm 9
\end{aligned}
$$

in the principal axes system of the molecule.

\section{Introduction}

The nuclear quadrupole interaction in molecules, due to the ${ }^{79} \mathrm{Br}$ and ${ }^{81} \mathrm{Br}$ nuclei with spins of $3 / 2$, causes each rotational transition to split into a number of components. These hyperfine splittings will yield information about the electronic environment of the bromine nucleus. In this way, the double-bond character of the $\mathrm{C}-\mathrm{Br}$ bond can be estimated.

Usually, the nuclear quadrupole coupling effects are calculated by means of a perturbation treatment. Large nuclear quadrupole moments may require treatment of the second order or higher. For molecules containing a bromine nucleus, a second-order treatment is usually not only necessary, but also sufficient. Except for a few lines, this also proved to be the case in 2-bromothiophene.

Besides the quadrupole interaction, 2-bromothiophene (Fig. 1) is also interesting from a structural point of view. In order to investigate ring-deformation effects, Harshbarger and Bauer ${ }^{1,2}$ have studied

Reprint requests to P. J. Mjöberg, Department of Physical Chemistry, The Royal Institute of Technology, S-100 44 Stockholm 70, Sweden.

$$
\begin{array}{r}
493.7 \pm 1.5, \\
-245.6 \pm 0.7, \\
-248.1 \pm 1.6, \\
64 \pm 8,
\end{array}
$$

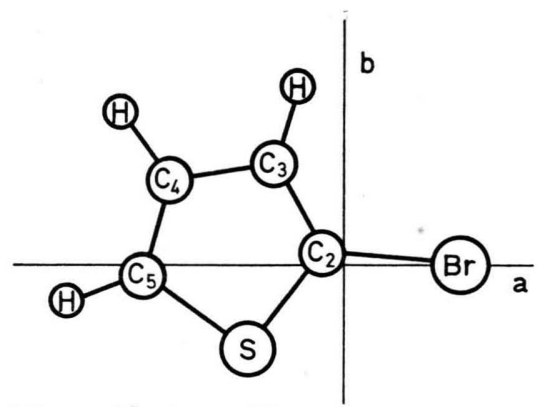

Fig. 1. 2-Bromothiophene. The orientation of the $a$ and $b$ principal axes is indicated.

2-chloro- and 2-bromothiophene by electron diffraction in the gas phase. In both cases, they found that two different structures, $\mathrm{A}$ and $\mathrm{B}$, could be equally well fitted to the experimental data. Neither of these structures showed a $\mathrm{C}_{2 v}$ symmetry of the ring.

However, by a recalculation from the data of Harshbarger and Bauer, Derissen et al. ${ }^{3}$ have found that a $\mathrm{C}_{2 v}$ ring structure or even a thiophene-like ring fit the experimental data just as well as the unsymmetric structures of Harshbarger and Bauer.

For comparison, the parameters and the corresponding calculated rotational constants of the different structures are shown in Table 1. 
Table 1. A comparison of different structures of 2 -bromothiophene $\left({ }^{81} \mathrm{Br}\right)$.

\begin{tabular}{|c|c|c|c|c|c|c|c|}
\hline & $\begin{array}{l}\text { Model A } \\
\text { of } \mathrm{H} \& \mathrm{~B} \text { a }\end{array}$ & $\begin{array}{l}\text { Model B } \\
\text { of } \mathrm{H} \& \mathrm{~B} \text { a }\end{array}$ & $\mathrm{C}_{2 v}$ ring b & $\begin{array}{l}\mathrm{C}_{2} v \text { ring, } \\
1 \text { restraint } \mathrm{b}\end{array}$ & $\begin{array}{l}\text { Model A } \\
\text { refined c }\end{array}$ & $\begin{array}{l}\text { From } \\
\text { thiophene d }\end{array}$ & $\begin{array}{l}\text { From } \\
\text { thiophene e }\end{array}$ \\
\hline $\mathrm{C}_{2}-\mathrm{S}(\AA)$ & 1.744 & 1.668 & 1.704 & 1.703 & 1.736 & 1.7140 & 1.714 \\
\hline $\mathrm{C}_{5}-\mathrm{S}$ & 1.668 & 1.740 & 1.704 & 1.703 & 1.672 & 1.7140 & 1.714 \\
\hline $\mathrm{C}_{2}-\mathrm{C}_{3}$ & 1.390 & 1.465 & 1.365 & 1.383 & 1.385 & 1.3696 & 1.370 \\
\hline $\mathrm{C}_{3}-\mathrm{C}_{4}$ & 1.467 & 1.396 & 1.455 & 1.418 & 1.463 & 1.4236 & 1.419 \\
\hline $\mathrm{C}_{4}-\mathrm{C}_{5}$ & 1.341 & 1.339 & 1.365 & 1.383 & 1.343 & 1.3696 & 1.370 \\
\hline $\mathrm{C}-\mathrm{H}$ & 1.091 & 1.089 & 1.078 & 1.093 & 1.100 & $\begin{array}{l}1.0776 \\
1.0805\end{array}$ & 1.092 \\
\hline $\mathrm{C}_{2}-\mathrm{Br}$ & 1.849 & 1.850 & 1.866 & 1.865 & 1.857 & 1.850 & 1.850 \\
\hline $\mathrm{C}_{2}-\mathrm{S}-\mathrm{C}_{5}\left({ }^{\circ}\right)$ & 92.0 & 92.8 & 91.9 & 92.3 & 91.9 & 92.17 & 92.2 \\
\hline $\mathrm{S}-\mathrm{C}_{5}-\mathrm{C}_{4}$ & 114.7 & 111.1 & 112.7 & 111.8 & 114.3 & 111.47 & 111.4 \\
\hline $\mathrm{S}-\mathrm{C}_{2}-\mathrm{C}_{3}$ & 110.9 & 111.1 & 112.7 & 111.8 & 111.4 & 111.47 & 111.4 \\
\hline $\mathrm{S}-\mathrm{C}_{2}-\mathrm{Br}$ & 120.7 & 125.2 & 121.5 & 122.5 & 120.6 & 119.85 & 119.85 \\
\hline $\mathrm{C}_{2}-\mathrm{C}_{3}-\mathrm{H}$ & 124.3 & 124.6 & 120 & 130 & 124.4 & 123.28 & 123.28 \\
\hline $\mathrm{S}-\mathrm{C}_{5}-\mathrm{H}$ & 118.3 & 120.0 & 117 & 126 & 118.5 & 119.85 & 119.85 \\
\hline $\mathrm{C}_{5}-\mathrm{C}_{4}-\mathrm{H}$ & 123.6 & 121.9 & 120 & 130 & 123.5 & 123.28 & 123.28 \\
\hline$A(\mathrm{MHz})$ & 5440.69 & 5417.30 & 5485.32 & 5407.55 & 5443.63 & 5478.98 & 5475.32 \\
\hline$B$ & 1121.72 & 1122.14 & 1125.45 & 1123.39 & 1121.65 & 1133.89 & 1133.42 \\
\hline$C$ & 929.98 & 929.59 & 938.85 & 930.15 & 930.02 & 939.46 & 939.04 \\
\hline
\end{tabular}

a $\mathrm{H} \& \mathrm{~B}=$ Harshbarger and Bauer ${ }^{2}$. The $\mathrm{H}$-angles were not determined by H\&B but were chosen by analogy with the thiophene structure determined by Bak et alias ${ }^{4}$.

a Determined by Derissen et alias ${ }^{3}$.

c Determined by Derissen et alias ${ }^{3}$. However, they did not specify the H-angles. These were chosen by analogy with the thiophene structure determined by Bak et alias ${ }^{4}$.

$d$ The thiophene structure was determined by Bak et al. ${ }^{4}$ from microwave spectroscopy.

e The thiophene structure was determined by Bonham and Momany ${ }^{5}$ from electron diffraction. However, they did not specify the $\mathrm{H}$-angles. These were chosen to be the same as in the thiophene structure of Bak et alias ${ }^{4}$.

The microwave spectra of the two ${ }^{35} \mathrm{Cl}$ and ${ }^{37} \mathrm{Cl}$ isotopic species of 2-chlorothiophene have already been reported ${ }^{6,7}$. The study of the microwave spectrum of 2-bromothiophene was undertaken mainly in order to investigate the quadrupole coupling effects in the spectrum due to the presence of the bromine nuclei, ${ }^{79} \mathrm{Br}$ and ${ }^{81} \mathrm{Br}$.

\section{Experimental}

The sample of 2-bromothiophene was purchased from E. Merck AG and was used without further purification.

The microwave spectrum was observed in the region 18000 to $40000 \mathrm{MHz}$ on a Hewlett-Packard $8460 \mathrm{~A}$ microwave spectrometer equipped with a phase-stabilized source oscillator and with Stark modulation at $33.33 \mathrm{kHz}$. The measurements were performed both at room temperature and at $-20^{\circ} \mathrm{C}$, at pressures ranging from 10 to $40 \mathrm{mT}$ Torr.

\section{Microwave Spectra and Rotational Constants}

2-Bromothiophene is a near-prolate asymmetric top $(x=-0.91)$ with the major component of the dipole moment along the $a$ principal axis.
The spectrum observed consists of $a$-type Rbranch transitions with $\Delta K_{-1}=0$ and $\Delta K_{+1}=1$ and with $\Delta F=+1$. The assignment of these lines was facilitated by their characteristic hyperfine splittings due to the quadrupole interaction. The asymmetry splitting of the $K_{-1}=1$ lines amounts to $1000-2000 \mathrm{MHz}$. The ground state rotational transitions of both isotopic species are listed in Table 2.

In the fitting procedure described in the next chapter, the rotational and centrifugal distortion constants were fitted simultaneously with the elements of the $\chi$-tensor in the inertial principal axes system. Table 3 shows the rotational constants, principal moments of inertia and centrifugal distortion coefficients. The large relative uncertainties in the centrifugal distortion constants may be anticipated for two different reasons. First, the centrifugal distortion correction amounts to only $0.1-1 \mathrm{MHz}$ for most of the lines and secondly, only ${ }^{a} R_{01}$-transitions were included in the fit.

We were not able to determine the dipole moment owing to the high $J$-values and low Stark effect of the unsplit lines in the spectrum. 
Table 2. Some rotational transitions $(\mathrm{MHz})$ of 2-bromothiophene in the ground state.

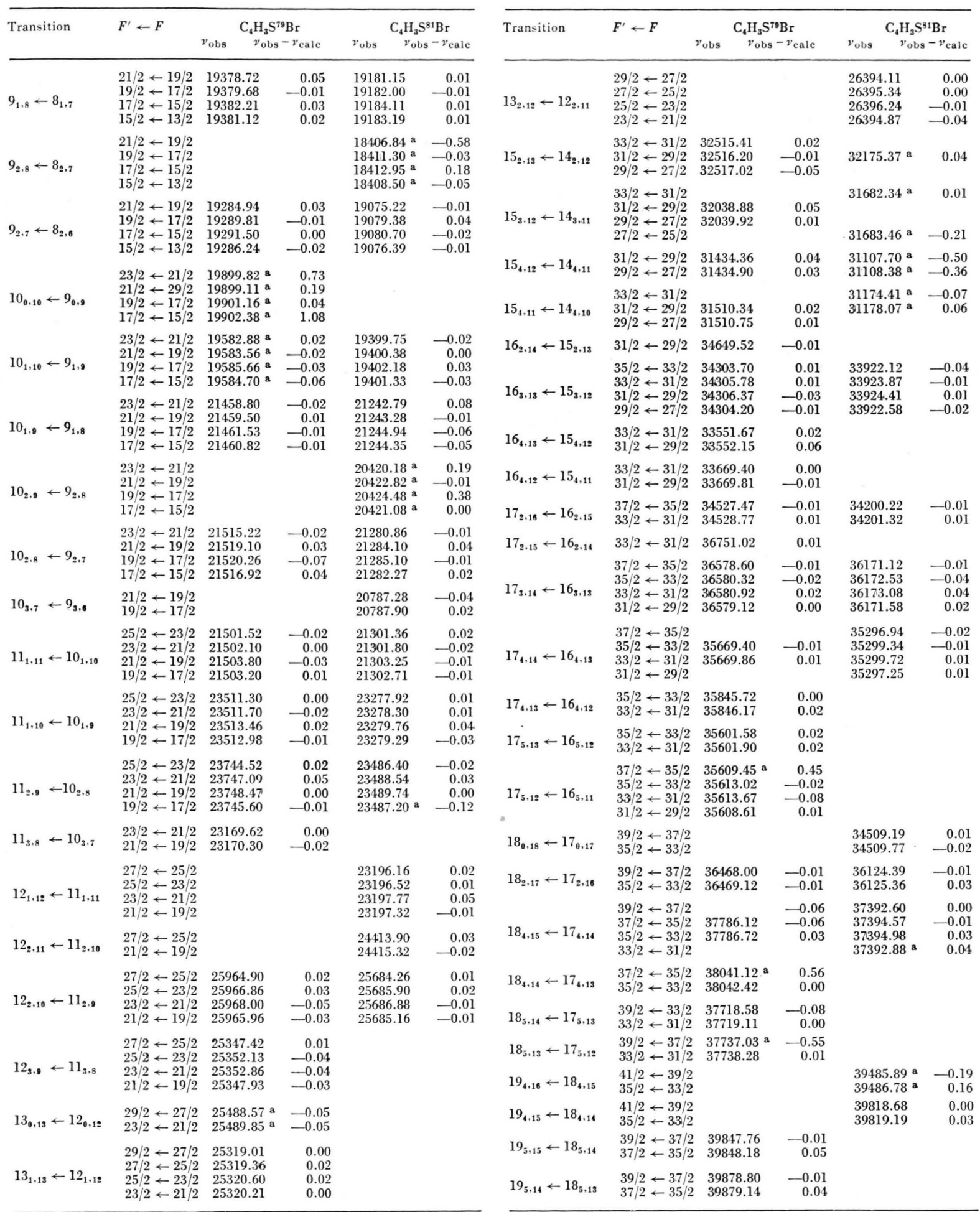

a These lines were not included in the final fit. 
Table 3. The rotational constants $(\mathrm{MHz})$, principal moments of inertia (amu $\AA^{2}$ ) and centrifugal distortion coef ficients $(\mathrm{Hz})$ of the two isotopic species of 2-bromothiophene.

\begin{tabular}{lcc}
\hline & $\mathrm{C}_{4} \mathrm{H}_{3} \mathrm{~S}^{79} \mathrm{Br}$ & $\mathrm{C}_{4} \mathrm{H}_{3} \mathrm{~S}^{81} \mathrm{Br}$ \\
\hline$A$ & $5403.432 \pm 0.111$ & $5403.563 \pm 0.094$ \\
$B$ & $1139.0689 \pm 0.0010$ & $1126.5173 \pm 0.0011$ \\
$C$ & $940.5142 \pm 0.0018$ & $931.9315 \pm 0.0009$ \\
$\varkappa$ & -0.911 & -0.913 \\
$I_{a}$ & $93.5289 \pm 0.0019$ & $93.5266 \pm 0.0016$ \\
$I_{b}$ & $443.6755 \pm 0.0004$ & $448.6189 \pm 0.0004$ \\
$I_{C}$ & $537.3412 \pm 0.0010$ & $542.2899 \pm 0.0005$ \\
$\Delta_{n} I_{c}-I_{a}-I_{b}$ & $0.1368 \pm 0.0022$ & $0.1444 \pm 0.0017$ \\
$\Delta_{J}$ & $52.0 \pm 5.1$ & $47.9 \pm 2.0$ \\
$\Delta_{J K}$ & $538 \pm 28$ & $434 \pm 52$ \\
$\Delta_{K}$ & $-1305 \pm 13004$ & $23833 \pm 15257$ \\
$\delta_{J}$ & $3.9 \pm 2.6$ & $10.0 \pm 0.9$ \\
$\delta_{K}$ & $865 \pm 428$ & $741 \pm 241$ \\
\hline
\end{tabular}

One vibrational satellite was observed for each of the bromine-isotopic species of the molecule. The rotational constants of these vibrational satellites are shown in Table 4, together with the calculated values of the vibration-rotation interaction constants.

Table 4. The rotational constants $(\mathrm{MHz})$, principal moments of inertia $\left(\mathrm{amu} \AA^{2}\right.$ ) of the first vibrational satellites together with the vibration-rotation interaction constants of 2-bromothiophene.

\begin{tabular}{lrr}
\hline & \multicolumn{1}{c}{$\mathrm{C}_{4} \mathrm{H}_{3} \mathrm{~S}^{79} \mathrm{Br}$} & \multicolumn{1}{c}{$\mathrm{C}_{4} \mathrm{H}_{3} \mathrm{~S}^{81} \mathrm{Br}$} \\
\hline$A$ & $5330.55 \pm 0.64$ & $5329.52 \pm 0.80$ \\
$B$ & $1139.961 \pm 0.006$ & $1127.401 \pm 0.008$ \\
$C$ & $941.578 \pm 0.006$ & $932.998 \pm 0.007$ \\
& & \\
$I_{a}$ & $94.808 \pm 0.011$ & $94.826 \pm 0.014$ \\
$I_{b}$ & $443.328 \pm 0.002$ & $448.267 \pm 0.003$ \\
$I_{c}$ & $536.734 \pm 0.003$ & $541.670 \pm 0.004$ \\
$\Delta_{=} I_{c}-I_{a}-I_{b}$ & $-1.402 \pm 0.012$ & $-1.423 \pm 0.015$ \\
$\alpha_{a}$ & $-72.88 \pm 0.65$ & $-74.04 \pm 0.81$ \\
$\alpha_{b}$ & $0.892 \pm 0.006$ & $0.884 \pm 0.008$ \\
$\alpha_{c}$ & $1.064 \pm 0.006$ & $1.066 \pm 0.007$ \\
\hline
\end{tabular}

The large negative change of the inertia defect in going from the ground to the vibrationally excited state is typical for an out-of-plane vibration ${ }^{8}$. Furthermore, since the $a$ principal axis of the molecule almost coincides with the $\mathrm{C}-\mathrm{Br}$ bond, the large negative value of the spectroscopic vibrationrotation interaction constant $\alpha_{a}$ in contrast to $\alpha_{b}$ and $\alpha_{c}$ indicates that the vibration is probably an out-ofplane $\mathrm{C}-\mathrm{Br}$ bending vibration. An estimate of the intensity ratio of the vibrational satellite to the ground state at room temperature yielded a value of 0.418 . This corresponds to an energy difference of $180 \mathrm{~cm}^{-1}$.

The variation of the inertia defect in going from the ground to the vibrationally excited state can also provide a certain amount of information about the character of the out-of-plane vibration. In the limiting case where the out-of-plane mode has a much higher frequency than any in-plane vibrational mode with which it may couple, Hanyu et al. ${ }^{9}$ have shown that

$$
\Delta_{v+1}-\Delta_{v}=-h / 2 \pi^{2} c \omega_{\mathrm{t}} .
$$

Inserting the values of the inertial defects of the states $v=0$ and $v=1$, we obtain the fundamental out-of-plane vibration frequency $\omega_{\mathrm{t}}=43 \mathrm{~cm}^{-1}$. The deviation from the value $180 \mathrm{~cm}^{-1}$ calculated above is large. This indicates a strong interaction between the out-of-plane vibration and low-frequency, inplane vibrations.

These results show a qualitative agreement with the results of infrared and Raman spectroscopic studies ${ }^{10-12}$. According to these studies, there is one out-of-plane vibration involving the bromine atom at $196 \mathrm{~cm}^{-1}$ and one in-plane vibration, also involving the bromine atom, at $209 \mathrm{~cm}^{-1}$.

\section{Nuclear Quadrupole Coupling}

\section{Theory}

The perturbation series expression for the quadrupole coupling correction to a rigid rotor energy level can be written as

$$
\begin{aligned}
E_{Q} & =E_{Q}{ }^{(1)}+E_{Q}{ }^{(2)}+\ldots=\left\langle I J \tau F M_{F}\left|H_{Q}\right| I J \tau F M_{F}\right\rangle \\
& +\sum_{J^{\prime} \tau^{\prime}} \frac{\left\langle I J \tau F M_{F}\left|H_{Q}\right| I J^{\prime} \tau^{\prime} F M_{F}\right\rangle^{2}}{W_{J \tau}-W_{J^{\prime} \tau^{\prime}}}+\ldots
\end{aligned}
$$

An expression for the perturbation operator $H_{Q}$ has been derived by Casimir ${ }^{13}$. The summation need not be extended over $I, F$ and $M_{F}$, because the operator $H_{Q}$ is diagonal in these indices.

The first-order energy contribution my be expressed as ${ }^{14}$

$$
\begin{gathered}
\left\langle I J \tau F M_{F}\left|H_{Q}\right| I J \tau F M_{F}\right\rangle=\frac{2}{J(J+1)} f(I, J, F) \\
\cdot\left[\chi_{a a} \frac{\partial E}{\partial A}+\chi_{b b} \frac{\partial E}{\partial B}+\chi_{c c} \frac{\partial E}{\partial C}\right]
\end{gathered}
$$


where $\chi_{g g}=e Q\left(\partial^{2} V / \partial g^{2}\right)$. Here, $g=a, b$ or $c$ are the molecule-fixed principal axes of inertia, and $f(I, J, F)$ is the Casimir function. Usually this expression is a good approximation to the quadrupole energy. However, if the matrix elements of the second-order terms are large and/or the rotational energies $W_{J \tau}$ and $W_{J^{\prime} \tau^{\prime}}$ are nearly degenerate, the higher-order correction must be included.

The matrix elements necessary for a second-order perturbation treatment of asymmetric-top molecules have been derived by Bragg ${ }^{15}$ :

$$
\begin{aligned}
& \left\langle I J \tau F M_{F}\left|H_{Q}\right| I J^{\prime} \tau^{\prime} F M_{F}\right\rangle \\
& \quad=g_{n}(I, J, F)\left\langle J \tau M_{J}=J\left|\chi_{Z Z}\right| J+n \tau^{\prime} M_{J}^{\prime}=J\right\rangle
\end{aligned}
$$

where $\chi_{Z Z}=e Q\left(\partial^{2} V / \partial Z^{2}\right)$, with $e Q$ being the quadrupole moment of the nucleus and $Z$ a spacefixed axis, while $n$ may be equal to 0,1 or 2 . The functions $g_{n}$ have the following explicit forms:

$$
\begin{aligned}
& g_{0}= {[3 C(C+1)-4 I(I+1) J(J+1)] } \\
& \times\{8 I(2 I-1) J(2 J-1)\}^{-1} \\
& g_{1}= {[F(F+1)-I(I+1)-J(J+2)] } \\
& \times[(I+J+F+2)(I-J+F)(J-I+F+1) \\
&\times(J+I-F+1)]^{1 / 2}\left\{8 I(2 I-1) J[(2 J+1)]^{1 / 2}\right\}^{-1} \\
& g_{2}= {[(I+J+F+2)(I+J+F+3)} \\
& \times(I-J+F-1)(I-J+F) \\
& \times(J-I+F+1)(J-I+F+2) \\
&\times(I+J-F+1)(I+J-F+2)]^{1 / 2} \\
& \times\left\{16 I(2 I-1)[(2 J+1)(J+1)]^{1 / 2}\right\}^{-1}
\end{aligned}
$$

where $C=F(F+1)-I(I+1)-J(J+1)$. Equations (4) and (5) will also yield the $\langle J \mid J-1\rangle$ and $\langle J \mid J-2\rangle$ matrix elements because of the Hermitian property of the operator $H_{Q}$.

The tensor component $\chi_{z Z}$ is related to the electric field gradients along the molecular principal axes, as well as to the direction cosines between the space-fixed $Z$ axis and the molecule-fixed principal axes. In the case of a planar near-prolate top, the following equation holds ${ }^{16}$

$$
\begin{gathered}
\chi_{Z Z}=\frac{1}{2}\left[\left(3 \Phi_{\left.\left.Z a^{2}-1\right) \chi_{a a}+\left(\Phi_{Z b}{ }^{2}-\Phi_{Z c}{ }^{2}\right) \varepsilon\right]}+2 \Phi_{Z a} \Phi_{Z b} \chi_{a b}\right.\right.
\end{gathered}
$$

where $\varepsilon=\chi_{b b}-\chi_{c c}$. In the planar case, $\chi_{b c}$ and $\chi_{c a}$ are equal to zero due to the symmetry of the molecule.

The evaluation of the matrix elements of Eq. (6) for asymmetric tops may be simplified by the use of group theory. The asymmetric top wave functions as well as the direction cosines appearing in Eq. (6) belong to the representations of the Four Group $\mathrm{V}^{15,17}$. Table 5 gives the species and characters of the $\mathrm{V}$ group, together with the symmetry classification of the direction cosines and their products and of the asymmetric rotor states. Thus, a matrix element of $\chi_{z Z}$ will vanish unless the direct product of

Table 5. Characters and species for the Four Group (V) and symmetry of the direction-cosine factors and the wave functions.

\begin{tabular}{llllllrr}
\hline Direction cosines & $K_{-1}$ & $K_{1}$ & $\begin{array}{l}\text { Spe- } \\
\text { cies }\end{array}$ & $E$ & $C_{\mathbf{2}}{ }^{a}$ & $C_{\mathbf{2}}{ }^{b}$ & $C_{\mathbf{2}}{ }^{c}$ \\
\hline$\Phi_{\mathrm{Z} a^{2}}, \Phi_{\mathrm{Z} b^{2}}, \Phi_{\mathrm{Zc}}{ }^{2}$ & $e$ & $e$ & $A$ & 1 & 1 & 1 & 1 \\
$\Phi_{\mathrm{Z} c} \Phi_{\mathrm{Z} b}, \Phi_{\mathrm{Z} a}$ & $e$ & $o$ & $B_{a}$ & 1 & 1 & -1 & -1 \\
$\Phi_{\mathrm{Z} c} \Phi_{\mathrm{Z} a}, \Phi_{\mathrm{Z} b}$ & $o$ & $o$ & $B_{b}$ & 1 & -1 & 1 & -1 \\
$\Phi_{\mathrm{Z} a} \Phi_{\mathrm{Z} b}, \Phi_{c}$ & $o$ & $e$ & $B_{c}$ & 1 & -1 & -1 & 1 \\
\hline
\end{tabular}

the two states $J \tau$ and $J^{\prime} \tau^{\prime}$ belongs to the same representation as $\Phi_{Z g} \Phi_{Z g^{\prime}}$. It is therefore obvious that two states may couple through either the first term of the right side of Eq. (6) or through the second term. The nearly degenerate states of identical $K_{-1}$ in near-prolate tops cannot couple through either term. Since the element $\chi_{a b}$ does not contribute to the first-order corrections, it can be determined accurately only in the case of accidental near degeneracies between states of appropriate symmetries and values of $J$.

A procedure for calculating the matrix elements in the Wang symmetric rotor wave functions of products of direction cosines has been described by Schwendeman ${ }^{18}$. Explicit expressions for all elements are, however, advantageous from many points of view. For this reason the matrix elements in the symmetric top wave functions have been derived. These elements are listed in Table 6. From this, the transformation to the Wang basis is simple.

The rotational wave functions for a slightly asymmetric top molecule may be expressed as linear combinations of Wang's symmetric top wave functions. The expression for Wang's functions is ${ }^{17}$

$$
\begin{aligned}
S_{J K M \gamma} & =\frac{1}{2}\left[\psi_{J K M}^{\times}+(-1)^{\gamma} \psi_{J-K M}^{\times}\right] \text {for } K \neq 0 \\
S_{J 0 M 0} & =\psi_{J O M}^{\times} \text {for } K=0
\end{aligned}
$$

where $\gamma$ is 0 or 1 and $\psi_{J K M}^{\times}=(-1)^{\beta} \psi_{J K M}$ with $\beta=\frac{1}{2}|K+M|+\frac{1}{2}|K-M|$. Then, up to the second order for the asymmetric top, we have

$$
\begin{gathered}
\psi_{K}=\left[1-\frac{\alpha^{2}+\alpha^{\prime 2}}{2}\right] S_{K}+\alpha S_{K-2}+\alpha^{\prime} S_{K+2} \\
+\beta S_{K-4}+\beta^{\prime} S_{K+4}
\end{gathered}
$$




\begin{tabular}{|c|c|c|c|}
\hline Operator & $J^{\prime}$ & $K^{\prime}$ & $\begin{array}{l}\text { Value of the matrix element } \\
\left\langle J K M_{J}=J|O p| J^{\prime} \cdot K^{\prime} M_{J^{\prime}}=J\right\rangle\end{array}$ \\
\hline \multirow{2}{*}{$\Phi_{Z a^{2}}$} & \multirow{2}{*}{$J+1$} & \multirow{2}{*}{$K$} & $2 K\left[(J+1)^{2}-K^{2}\right]^{1 / 2}$ \\
\hline & & & $(J+1)(J+2)(2 J+3)^{1 / 2}$ \\
\hline \multirow{2}{*}{$\Phi_{Z a^{2}}$} & \multirow{2}{*}{$J+2$} & \multirow{2}{*}{$K$} & $2\left[(J+1)^{2}-K^{2}\right]^{1 / 2}\left[(J+2)^{2}-K^{2}\right]^{1 / 2}$ \\
\hline & & & $(J+2)(2 J+3)[(J+1)(2 J+5)]^{1 / 2}$ \\
\hline \multirow{2}{*}{$\Phi_{Z b^{2}}$} & \multirow{2}{*}{$J$} & \multirow{2}{*}{$K-2$} & {$[(J-K+1)(J-K+2)(J+K-1)(J+K)]^{1 / 2}$} \\
\hline & & & $2(J+1)(2 J+3)$ \\
\hline \multirow{2}{*}{$\Phi_{Z b^{2}}$} & \multirow{2}{*}{$J$} & \multirow{2}{*}{$K+2$} & {$[(J-K-1)(J-K)(J+K+1)(J+K+2)]^{1 / 2}$} \\
\hline & & & $2(J+1)(2 J+3)$ \\
\hline$\Phi_{Z b^{2}}$ & $J+1$ & $K-2$ & {$[(J-K+1)(J-K+2)(J-K+3)(J+K)]^{1 / 2}$} \\
\hline \multirow{2}{*}{$\Phi_{Z b^{2}}$} & \multirow{2}{*}{$J+1$} & \multirow{2}{*}{$K$} & $\begin{array}{l}2(J+1)(J+2)(2 J+3)^{1 / 2} \\
-K\left[(J+1)^{2}-K^{2}\right]^{1 / 2}\end{array}$ \\
\hline & & & $(J+1)(J+2)(2 J+3)^{1 / 2}$ \\
\hline \multirow{2}{*}{$\Phi_{Z b^{2}}$} & \multirow{2}{*}{$J+1$} & & $-[(J-K)(J+K+1)(J+K+2)(J+K+3)]^{1 / 2}$ \\
\hline & & NT2 & $2(J+1)(J+2)(2 J+3)^{\frac{1}{1 / 2}}$ \\
\hline$\Phi_{Z b^{2}}$ & $J+2$ & $K-2$ & {$[(J-K+1)(J-K+2)(J-K+3)(J-K+4)]^{1 / 2}$} \\
\hline & & & $\begin{array}{c}2(J+2)(2 J+3)[(J+1)(2 J+5)]^{1 / 2} \\
-\left[(J+1)^{2}-K^{2}\right]^{1 / 2}\left[(J+2)^{2}-K^{2}\right]^{1 / 2}\end{array}$ \\
\hline$\Phi_{Z b^{2}}$ & $J+2$ & $K$ & $(J+2)(2 J+3)[(J+1)(2 J+5)]^{1 / 2}$ \\
\hline$\Phi_{Z b^{2}}$ & $J+2$ & $K+2$ & {$[(J+K+1)(J+K+2)(J+K+3)(J+K+4)]^{1 / 2}$} \\
\hline & $j+2$ & $\Lambda+2$ & $2(J+2)(2 J+3)[(J+1)(2 J+5)]^{1 / 2}$ \\
\hline$\Phi_{Z c^{2}}$ & $J$ & $K-2$ & $-[(J-K+1)(J-K+2)(J+K-1)(J+K)]^{1 / 2}$ \\
\hline & & & $2(J+1)(2 J+3)$ \\
\hline$\Phi_{Z c^{2}}$ & $J$ & $K+2$ & $-[(J-K-1)(J-K)(J+K+1)(J+K+2)]^{1 / 2}$ \\
\hline & & & $2(J+1)(2 J+3)$ \\
\hline$\Phi_{Z c^{2}}$ & $J+1$ & $K-2$ & $-[(J-K+1)(J-K+2)(J-K+3)(J+K)]^{1 / 2}$ \\
\hline & & & $\begin{array}{l}2(J+1)(J+2)(2 J+3)^{1 / 2} \\
-K\left[(J+1)^{2}-K^{2}\right]^{1 / 2}\end{array}$ \\
\hline$\Phi_{Z c^{2}}$ & $J+1$ & $K$ & $(J+1)(J+2)(2 J+3)^{1 / 2}$ \\
\hline$\Phi_{\mathrm{Z} c^{2}}$ & $J+1$ & $K+2$ & {$[(J-K)(J+K+1)(J+K+2)(J+K+3)]^{1 / 2}$} \\
\hline & & NT2 & $2(J+1)(J+2)(2 J+3)^{1 / 2}$ \\
\hline$\Phi_{Z c^{2}}$ & $J+2$ & $K-2$ & $-[(J-K+1)(J-K+2)(J-K+3)(J-K+4)]^{1 / 2}$ \\
\hline & & & $\begin{array}{l}2(J+2)(2 J+3)[(J+1)(2 J+5)]^{1 / 2} \\
-\left[(J+1)^{2}-K^{2}\right]^{1 / 2:}\left[(J+2)^{2}-K^{2}\right]^{1 / 2}\end{array}$ \\
\hline$\Phi_{Z c^{2}}$ & $J+2$ & $K$ & $(J+2)(2 J+3)[(2 J+1)(2 J+5)]^{1 / 2}$ \\
\hline$\Phi_{Z c^{2}}$ & $J+2$ & $K+2$ & $-[(J+K+1)(J+K+2)(J+K+3)(J+K+4)]^{1 / 2}$ \\
\hline & $5+2$ & $1+2$ & $2(J+2)(2 J+3)[(J+1)(2 J+5)]^{1 / 2}$ \\
\hline$\Phi_{Z b^{2}}-\Phi_{Z c^{2}}$ & $J$ & $K-2$ & {$[(J-K+1)(J-K+2)(J+K-1)(J+K)]^{1 / 2}$} \\
\hline & & & 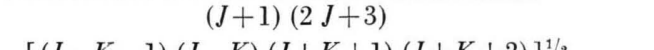 \\
\hline$\Phi_{Z b^{2}}-\Phi_{Z c^{2}}$ & $J$ & $K+2$ & $\frac{[(J-K-1)(J-K)(J+K+1)(J+K+2)]^{1 / 2}}{(J+1)(2 J+3)}$ \\
\hline$\Phi_{Z b^{2}}-\Phi_{Z c^{2}}$ & & & {$[(J-K+1)(J-K+2)(J-K+3)(J+K)]^{1 / 2}$} \\
\hline & $J+1$ & $K-2$ & $(J+1)(J+2)(2 J+3)^{1 / 2}$ \\
\hline$\Phi_{Z b^{2}}-\Phi_{Z c^{2}}$ & $J+1$ & $K+2$ & $-[(J-K)(J+K+1)(J+K+2)(J+K+3)]^{1 / 2}$ \\
\hline & & & $(J+1)(J+2)(2 J+3)^{1 / 2}$ \\
\hline$\Phi_{Z b^{2}}-\Phi_{Z c^{2}}$ & $J+2$ & $K-2$ & {$[(J-K+1)(J-K+2)(J-K+3)(J-K+4)]^{1 / 2}$} \\
\hline & & & $\begin{array}{c}(J+2)(2 J+3)[(J+1)(2 J+5)]^{1 / 2} \\
{[(J+K+1)(J+K+2)(J+K+2)(J+K+4)]^{1 / 2}}\end{array}$ \\
\hline$\Phi_{Z b^{2}}-\Phi_{Z c^{2}}$ & $J+2$ & $K+2$ & $(J+2)(2 J+3)[(J+1)(2 J+5)]^{1 / 2}$ \\
\hline$\Phi_{Z b} \Phi_{Z a}$ & $J$ & $K-1$ & $(2 K-1)[(J-K+1)(J+K)]^{1 / 2}$ \\
\hline & & & $2(J+1)(2 J+3)$ \\
\hline$\Phi_{Z b} \Phi_{Z a}$ & $J$ & $K+1$ & $(2 K+1)[(J-K)(J+K+1)]^{1 / 2}$ \\
\hline & & & $2(J+1)(2 J+3)$ \\
\hline$\Phi_{Z b} \Phi_{Z a}$ & $J+1$ & $K-1$ & $\frac{(J+2 K)[(J-K+1)(J-K+2)]^{1 / 2}}{2(J+1)(J+2)(2 J+3)^{1 / 2}}$ \\
\hline$\Phi_{Z b} \Phi_{Z a}$ & $J+1$ & $K+1$ & $(J-2 K)[(J+K+1)(J+K+2)]^{1 / 2}$ \\
\hline$\$ 20 \times 2 a$ & $J+1$ & $\Lambda+1$ & $2(J+1)(J+2)(2 J+3)^{1 / 2}$ \\
\hline$\Phi_{Z b} \Phi_{Z a}$ & $J+2$ & $K-1$ & {$\left[(J+1)^{2}-K^{2}\right]^{1 / 2}[(J-K+2)(J-K+3)]^{1 / 2}$} \\
\hline & & & $(J+2)(2 J+3)[(J+1)(2 J+5)]^{1 / 2}$ \\
\hline$\Phi_{Z b} \Phi_{Z a}$ & $J+2$ & $K+1$ & $\frac{-\left[(J+1)^{2}-K^{2}\right]^{1 / 2}[(J+K+2)(J+K+3)]^{1 / 2}}{(J+2)(2 J+3)[(J+1)(2 J+5)]^{1 / 2}}$ \\
\hline & & & $(J+2)(2 J+3)[(J+1)(2 J+5)]^{1 / 2}$ \\
\hline
\end{tabular}

Table 6. Values of matrix elements of some products of direction cosines in a symmetric rotor basis a.

a These matrix elements include an arbitrary choice of phase. This phase choice is identical to that of Cross et al. ${ }^{19}$ and Shwendeman ${ }^{18}$. 
where the $J, M$ and $\gamma$ labels have been omitted. The coefficients $\alpha, \alpha^{\prime}, \beta$ and $\beta^{\prime}$ have been given by Lide $^{20}$. In the present case, however, the zeroth order function

$$
\psi_{K}=S_{K}
$$

proved sufficient.

It may be pointed out that an alternative procedure for calculating the matrix elements in the second-order terms has been described by Ferguson ${ }^{21}$.

\section{Spectrum}

First-order perturbation theory predicts that for transitions having $J>3$, the four hyperfine components will appear as two doublets with equal splitting. However, it was immediately observed from the microwave spectrum that the splitting was unsymmetrical. From this it was evident that the perturbation treatment would have to be extended at least up to and including second order in the analysis of the quadrupole coupling effects.

At first, a simplified procedure suggested by Mirri and Caminati ${ }^{22}$ was tried. In this procedure the expression for the nuclear quadrupole coupling constant $\chi_{z z}$ in Eq. (6) is simplified to

$$
\chi_{Z Z}=\frac{1}{2}\left(3 \Phi_{Z a}{ }^{2}-1\right) \chi_{a a} .
$$

This method will generally suffice in cases of small values of $\chi_{a b}$ with no accidental degeneracies between states that couple through $\Phi_{Z a} \Phi_{Z b}$. Furthermore, the term with $\varepsilon=\chi_{b b}-\chi_{c c}$ in Eq. (6) is often insignificant. This is because the matrix elements of $\Phi_{Z b^{2}}{ }^{2}-\Phi_{Z c}{ }^{2}$ are of the same order as those of $\Phi_{Z a}{ }^{2}$, and because $\chi_{b b} \approx \chi_{c c}$ in many cases. By this procedure, an improved agreement between observed and calculated line-frequencies was obtained for some lines but not for others. Thus, the more complete theory outlined in the previous section had to be applied. It then became apparent that the inclusion of the tensorial component $\chi_{a b}$ was necessary in order to correctly calculate many lines.

The fitting was accomplished by an iterative procedure. First, the spectrum was fitted by firstorder theory using a conventional method of least squares. The constants thus obtained were then used to calculate a rough value of $\chi_{a b}$, assuming that the $\mathrm{C}-\mathrm{Br}$ axis is a principal axis of the tensor. Next, the calculated second-order contribution was subtracted from the observed line-frequencies and another first-order fit could be carried out. In the following steps of the iteration an improved value of $\chi_{a b}$ was obtained by applying the second-order theory to some transitions showing a particularly large second-order effect. After fitting $\chi_{a b}$ in this way and deducing the second-order effects another first order fit was carried out. The described iterative procedure was performed three times.

In view of the approximate nature of the perturbation treatment, it was considered advisable not to include the transitions with the largest secondorder contributions in the final first-order fit. For some of these lines, the calculated second-order perturbation could not fully account for the difference between the observed frequencies and the first-order calculations. This disagreement might partly be due to the need of a better wave function and partly to the need of still higher perturbation treatments. The latter factor is probably the dominant one e.g. in the cases of transitions involving either the level $10_{3,8}$ or the level $11_{2.10}$ of $\mathrm{C}_{4} \mathrm{H}_{3} \mathrm{~S}^{79} \mathrm{Br}$. These levels are only about $14 \mathrm{MHz}$ apart, giving rise to large coefficients of $\chi_{a b}$ in the second-order sum.

The quadrupole coupling constants obtained are listed in Table 7. It should be observed that the sign of the off-diagonal element $\chi_{a b}$ cannot be obtained from the analysis of the second-order contri-

Table 7. Nuclear quadrupole coupling constants $(\mathrm{MHz})$ of the bromine atom in 2-bromothiophene.

\begin{tabular}{lcc}
\hline & $\mathrm{C}_{4} \mathrm{H}_{3} \mathrm{~S}^{79} \mathrm{Br}$ & $\mathrm{C}_{4} \mathrm{H}_{3} \mathrm{~S}^{81} \mathrm{Br}$ \\
\hline$\chi_{a a}$ & $592.7 \pm 1.5$ & $493.7 \pm 1.5$ \\
$\chi_{b b}$. & $-295.3 \pm 0.6$ & $-245.6 \pm 0.7$ \\
$\chi_{c c}$ & $-297.4 \pm 1.6$ & $-248.1 \pm 1.6$ \\
$\chi_{a b}{ }^{\mathrm{a}}$ & $80 \pm 9$ & $64 \pm 8$ \\
$\chi_{x x}$ & $-302.4 \pm 2.7$ & $-251.1 \pm 2.6$ \\
$\chi_{y y}$ & $-297.4 \pm 1.6$ & $-248.1 \pm 1.6$ \\
$\chi_{z z}$ & $599.8 \pm 2.2$ & $499.2 \pm 2.0$ \\
$\theta_{z a}\left({ }^{\circ}\right)$ & $5.11 \pm 0.56$ & $4.91 \pm 0.60$ \\
$\theta \mathrm{b}\left({ }^{\circ}\right)$ & & 3.69 to 6.90 \\
$\Delta$ & $-5.0 \pm 3.2$ & $-3.0 \pm 3.0$ \\
$\eta$ & $-0.008 \pm 0.005$ & $-0.006 \pm 0.006$ \\
$\delta$ & $0.004 \pm 0.003$ & $0.003 \pm 0.003$ \\
\hline
\end{tabular}

a The sign of $\chi_{a b}$ could not be determined from the secondorder quadrupole effect alone.

b $\theta$ is the angle between the $\alpha$ inertial principal axis and the $\mathrm{C}-\mathrm{Br}$ internuclear line, calculated from the different structures in Table 1. 
butions alone. In Table 7, the principal elements $\chi_{x x}, \chi_{y y}$ and $\chi_{z z}$ of the $\chi$-tensor are also listed. These elements are obtained from the ones in the inertial principal axes system by diagonalization by a rotational operator

$$
T=\left(\begin{array}{ccc}
\cos \theta_{z a} & \sin \theta_{z a} & 0 \\
-\sin \theta_{z a} & \cos \theta_{z a} & 0 \\
0 & 0 & 1
\end{array}\right) .
$$

In accordance with current usage, let the $y$ principal axis of the field gradient tensor coincide with the $c$ axis of the inertial moment. Furthermore, let the $z$ principal axis be the one that is closest to the $\mathrm{C}-\mathrm{Br}$ internuclear line. From the diagonalization procedure, the angle $\theta_{z a}$ between the $z$ principal axis of the tensor and the $a$ inertial principal axis is thus obtained. For comparison we have also calculated the angle $\theta$ between the $a$ axis and the $\mathrm{C}-\mathrm{Br}$ bond direction from the different structures of Table 1. In Table 7 we have also listed some parameters of interest in the characterization of the $\mathrm{C}-\mathrm{Br}$ bond. The asymmetry parameters $\Delta$ and $\eta$ are defined according to ${ }^{23}$

$$
\Delta=\chi_{x x}-\chi_{y y}
$$

1 W. R. Harshbarger, Ph. D. Dissertation, Cornell University, September 1968.

2 W. R. Harshbarger and S. H. Bauer, Acta Cryst. B 26, 1010 [1970].

3 J. L. Derissen, J. W. M. Kocken, and R. H. van Weelden, Acta Cryst. B 27, 1692 [1971].

4 B. Bak, D. Christensen, L. Hansen-Nygaard, and J. Rastrup-Andersen, J. Mol. Spectrosc. 7, 58 [1961].

5 R. A. Bonham and F. A. Momany, J. Phys. Chem. 67, 2474 [1963].

6 J. Mjöberg and S. Ljunggren, Z. Naturforsch 28 a, 729 [1973].

7 R. T. Walden, Ph. D. Dissertation, Mississippi State University, August 1973.

8 D. R. Herschbach and V. W. Laurie, J. Chem. Phys. 40, 3142 [1964].

9 Y. Hanyu, C. O. Britt, and J. E. Boggs, J. Chem. Phys. 45, 4725 [1966].

10 M. Horak, I. J. Hyams, and E. R. Lippincott, Spectrochim. Acta 22, 1355 [1966].

11 J. J. Peron, P. Saumagne, and J. M. Lebas, Spectrochim. Acta 26 A, 1651 [1970]. and

$$
\eta=\left(\chi_{x x}-\chi_{y y}\right) / \chi_{z z} .
$$

The double-bond character, $\delta$, has been calculated from the relation suggested by Goldstein ${ }^{23}$

$$
\delta=\Delta / \Delta_{\text {atomic }}
$$

where $\Delta_{\text {atomic }}=\frac{3}{2} e Q q_{\text {atomic }}$. For $e Q q_{\text {atomic }}$, the values given by King and Jaccarino ${ }^{24}(-769.756$ $\mathrm{MHz}$ for ${ }^{79} \mathrm{Br}$ and $-643.033 \mathrm{MHz}$ for ${ }^{81} \mathrm{Br}$ ) were used. As appears from Table 7, the double-bond character in 2-bromothiophene is very small. This was also found to be the case for 2-chlorothiophene in which a value of $\delta=0.6 \%$ was found ${ }^{6}$.

\section{Acknowledgements}

We are much obliged to Dr. Hasse Karlsson for allowing us to perform our $K$-band measurements on the microwave spectrometer at the University of Göteborg. We are indebted to Mr. Claus Nielsen for valuable discussions. One of us (P. J. M.) is also very grateful to Prof. Eizi Hirota for valuable comments.

This work was supported by the Swedish Natural Science Research Council (NFR).

12 J. H. S. Green, Spectrochim. Acta 27 A, 2015 [1971].

13 H. B. G. Casimir, On the Interaction between Atomic Nuclei and Electrons, Prize Essay. Haarlem: Teyler's Tweede Gen. De Erven F. Bohn, N. V. 1936.

14 J. E. Wollrab, Rotational Spectra and Molecular Structure, Academic Press, New York 1967.

15 J. K. Bragg, Phys. Rev. 74, 533 [1948].

16 H. W. Morgan and J. H. Goldstein, J. Chem. Phys. 22, 1427 [1954].

17 G. W. King, R. M. Hainer, and P. C. Cross, J. Chem. Phys. 11, 27 [1943].

18 R. H. Schwendeman, J. Mol. Spectrosc. 7, 280 [1961].

19 P. C. Cross, R. M. Hainer, and G. W. King, J. Chem. Phys. 12, 210 [1944].

20 D. R. Lide, Jr., J. Chem. Phys. 20, 1761 [1952].

21 A. C. Ferguson, Ph. D. Dissertation, The University of Wisconsin, 1969.

22 A. M. Mirri and W. Caminati, Chem. Phys. Letters 8, 409 [1971].

23 J. H. Goldstein, J. Chem. Phys. 24, 106 [1956].

24 J. G. King and V. Jaccarino, Phys. Rev. 94, 1610 [1954]. 